\title{
Selective Synthesis of Benzaldehydes by Hypochlorite Oxidation of Benzyl Alcohols under Phase Transfer Catalysis
}

\author{
K. Bijudas ${ }^{*}$, P. Bashpa ${ }^{1}$, V. P. Bibin ${ }^{2}$, Lakshmi Nair ${ }^{2}$, A. P. Priya ${ }^{2}$, M. Aswathy ${ }^{2}$, \\ C. Krishnendu ${ }^{2}$, P. Lisha ${ }^{2}$ \\ ${ }^{1}$ Department of Chemistry, N. S. S. College, Manjeri, Malappuram, Kerala - 676122, India \\ ${ }_{2}^{2}$ Graduate Students, Department of Chemistry, N. S. S. College, Manjeri, Malappuram, Kerala - \\ 676122, India
}

Received: 10th August 2014; Revised: 20th September 2014; Accepted: 21st September 2014

\begin{abstract}
The selective synthesis of benazaldehyde and substituted benzaldehydes from corresponding benzyl alcohols has been carried out by using hypochlorite as an oxidant in organic medium under phase transfer catalysis. The reaction is highly selective since no traces of benzoic acid or substituted benzoic acids have been detected and the yield is found to be more than $90 \%$. The products obtained were precipitated as 2,4-dinitrophenylhydrazone and the recrystallised products were characterized by melting point and by spectrophotometric techniques like infra red and UV-Visible analysis. (C) 2015 BCREC UNDIP. All rights reserved.
\end{abstract}

Keywords: Selective synthesis; Hypochlorite; Benzyl alcohol; Substituted benzyl alcohols; Phase transfer catalysis

How to Cite: Bijudas, K., Bashpa, P., Bibin, V. P., Nair, L., Priya, A. P., Aswathy, M., Krishnendu, C., Lisha, P. (2015). Selective Synthesis of Benzaldehydes by Hypochlorite Oxidation of Benzyl Alcohols under Phase Transfer Catalysis. Bulletin of Chemical Reaction Engineering \& Catalysis, 10 (1): 38-42. (doi:10.9767/bcrec.10.1.7189.38-42)

Permalink/DOI: http://dx.doi.org/10.9767/bcrec.10.1.7189.38-42

\section{Introduction}

Oxidation reactions using different oxidizing agents are having great significance in synthesis of various organic compounds [1-2]. The introduction of phase transfer catalysis (PTC) has revolutonalised organic synthesis in respect of anion dissolution in non polar solvents with their ability to catalyse the reaction. PTC is one of the most efficient methods that can be used to carry out a variety of chemical reactions under mild conditions with improved re-

* Corresponding Author.

E-mail: bijudask@gmail.com (K. Bijudas)

Tel: +91 9447170209, Fax: +914832766202 sults. Adoption of PTC technique in industry provides cost reduction and pollution prevention which are highly significant in the era of green chemistry [3-5]. There are reports on using permanganate and chromate as oxidizing agent in organic synthesis under PTC [6-12] but reports on the use of hypochlorite as oxidant in synthetic preparation of organic compounds are scanty [13-17]. Hypochlorite is one of the most selective, mild oxidizing agents in organic synthesis. Hypochlorite is easily available, cheap and environmental friendly. The major use of hypochlorite in synthetic organic chemistry is in the oxidation of primary and secondary alcohols to corresponding aldehydes and ketones. 
Benzaldehyde is an important starting compound for the manufacture of various compounds like dyes, odorants, flavours, perfumes etc. Currently benzaldehyde is produced by the direct oxidation of toluene or by side chain chlorination of toluene followed by hydrolysis of benzal chloride. Both these methods have disadvantages like requirement of high temperature, low yield, formation of by-products etc. The use of phase transfer catalysis can overcome all these problems. The reaction will not occur or will be very slow without the use of phase transfer catalysts (PT catalysts) in both polar and non polar medium. Even though there are lots of reports on such type of oxidation in polar medium reports using phase transferred hypochlorite as a selective oxidant in organic solvents is scanty and this prompted us to carry out this work.

The present paper reports the selective oxidation of benzyl alcohol and substituted benzyl alcohols to corresponding benzaldehydes by using various quaternary ammonium and phosphonium salts as PT catalysts in various organic solvents like ethyl acetate and toluene by hypochlorite. PT catalyst transfers the hypochlorite ion as an ion pair from the aqueous phase to organic phase and the oxidation occurs there with ease.

\section{Materials and Methods}

\subsection{Materials}

$5 \%$ solution of sodium hypochlorite (NICE Chemicals, India) was used as such in the entire process. Benzyl alcohol (AR) was further purified by distillation under reduced pressure. The substituted benzyl alcohols, 4hydroxybenzyl alcohol, 3-hydroxybenzyl alcohol, 4-chlorobenzyl alcohol, 3-chlorobenzyl alcohol, 4-methoxybenzyl alcohol, 3-methoxybenzyl alcohol, 4-nitrobenzyl alcohol, 3-nitrobenzyl alcohol and 4-methylbenzyl alcohol (spectrochem, India Ltd. Mumbai and Merck KGaA, Germany) were used, such as tetrabutylammonium bromide (TBAB), tetrabutylammonium hydrogen sulphate (TBAHS), cetyltrimethylammonium bromide (CTMAB) (Spectrochem India Ltd. Mumbai), and tetrabutylphosphonium bromide (Merck KGaA, Germany), were used as PT catalyst. The organic solvents toluene and ethyl acetate were purified according to the standard procedure [18].

\subsection{Synthesis}

Synthetic analysis was carried out in heterogeneous manner. Benzyl alcohol and substi- tuted benzyl alcohols $(0.1 \mathrm{~mol})$ dissolved in 50 $\mathrm{ml}$ toluene or ethyl acetate which contains 0.01 mol PT catalyst was mixed with $50 \mathrm{ml}$ sodium hypochlorite. The mixture was stirred vigorously using a magnetic stirrer for about thirty minute at room temperature. The organic layer was extracted with ether three times. This organic layer was again extracted with $10 \%$ sodium bicarbonate and both organic and aqueous layers were separated. A saturated solution of 2,4-dinitrophenylhydrazine in $\mathrm{HCl}$ was added to the organic layer and kept overnight in refrigerator. The obtained product precipitated as 2,4-dinitrophenylhydrazone (DNP) was filtered off, recrystallised from ethanol, dried and weighed. The product obtained was analysed by its melting point and other qualitative analytical methods like UV-Visible spectrophotometer and IR spectrophotometer. The aqueous layer after extraction with sodium bicarbonate was acidified with concentrated $\mathrm{HCl}$ to detect the presence of acid.

\section{Results and Discussion}

Benzyl alcohol and substituted benzyl alcohols on oxidation under heterogeneous condition gave corresponding aldehyde as the product with very high yield (above 90\%). The oxidation reaction was carried out at room temperature and there was no further oxidation to acid. This is evident from the absence of benzoic acid or substituted benzoic acids in the reaction mixture. The recrystallised sample of products in the form of 2,4 - dinitrophenylhy-

Table 1. Melting point of products in the form of 2,4-dinitrophenylhydrazone on oxidation of benzyl alcohol and substituted benzyl alcohols

\begin{tabular}{clc}
\hline $\begin{array}{c}\text { Sl. } \\
\text { No. }\end{array}$ & \multicolumn{1}{c}{ Substrate } & $\begin{array}{c}\text { Melting } \\
\text { point }\left({ }^{\circ} \mathrm{C}\right)\end{array}$ \\
\hline 1 & Benzyl alcohol & $239 \pm 2$ \\
2 & 4-chlorobenzyl alcohol & $257 \pm 2$ \\
3 & 3-chlorobenzyl alcohol & $255 \pm 2$ \\
4 & 4-nitrobenzyl alcohol & $322 \pm 2$ \\
5 & 3-nitrobenzyl alcohol & $291 \pm 2$ \\
6 & 4-hydroxybenzyl alcohol & $268 \pm 2$ \\
7 & 3-hydroxybenzyl alcohol & $253 \pm 2$ \\
8 & 4-methylbenzyl alcohol & $232 \pm 2$ \\
9 & 4-methoxybenzyl alcohol & $253 \pm 2$ \\
10 & 3-methoxybenzyl alcohol & $255 \pm 2$ \\
\hline
\end{tabular}


drazone were characterized by its melting point and is given in Table 1.

The obtained results of melting point were compared with the values of authentic samples and found to have excellent similarities. This showed that the products obtained may be corresponding aldehyde. This was further confirmed by IR and UV- Visible spectral analysis by taking benzyl alcohol as the typical substrate.

The infra red absorption spectrum was recorded from $\mathrm{KBr}$ pellets using Jasco FT-IR 4100 spectrophotometer (Japan) and is shown in Figure 1. The IR spectrum showed sharp peaks at $3287 \mathrm{~cm}^{-1}\left(\mathrm{~N}-\mathrm{H}\right.$ stretching), $3090 \mathrm{~cm}^{-1}$ (Aromatic C-H stretching), $1744 \mathrm{~cm}^{-1}$ and 1620 $\mathrm{cm}^{-1}\left(\mathrm{C}=\mathrm{N}\right.$ stretching of hydrazone), $1585 \mathrm{~cm}^{-1}$ $(\mathrm{C}=\mathrm{C}$ stretching $), 1516 \mathrm{~cm}^{-1}\left(\mathrm{Ar}-\mathrm{NO}_{2}\right.$ asymmetric stretching), $1329 \mathrm{~cm}^{-1}\left(\mathrm{Ar}-\mathrm{NO}_{2}\right.$ symmetric stretching), and $1136 \mathrm{~cm}^{-1}$ (C-NH stretching). Presence of all these peaks leads to the conclusion that the product formed may be 2,4dinitrophenylhydrazone of benzaldehyde. Furthermore this spectrum was compared with the IR spectrum of 2,4-dinitrophenylhydrazone of pure benzaldehyde and found to have excellent similarities.

UV-Visible absorption of the product obtained on the oxidation of benzyl alcohol was recorded by Hitachi U-3000 UV-Visible spectrophotometer using $1 \mathrm{~cm}$ quartz cell and spectrograde ethanol (Merck, India) as solvent and is given in Figure 2. In the UV-Visible spectrum of 2,4-dinitrophenylhydrazone of the product on the oxidation of benzyl alcohol showed intense peaks at $235 \mathrm{~nm}$ and $353 \mathrm{~nm}$ and were assigned to $p-p^{*}$ and $n-p^{*}$ transition of the nitrogen containing aromatic compound. The UVVisible spectrum of the obtained product was compared with the UV-Visible spectrum 2, 4dinitrophenylhydrazone of pure benzaldehyde and gave excellent similarities.

All the above analyses, viz., melting point determination, IR and UV-Visible spectral studies showed that the product formed on the oxidation of benzyl alcohol and substituted benzyl alcohols by hypochlorite under phase transfer catalysis was corresponding benzaldehyde. The reaction was found to be very smooth in both the solvents, namely ethyl acetate and toluene. But the yield of benzaldehyde and ease of reaction was found to be more in ethyl acetate than that in toluene. This may be due to the fact that ethyl acetate is more polar than toluene. Solubility and partitioning of quaternary salts are increased by increase in the polarity of the organic phase.

All the four catalysts were found to be very effective in the oxidation reaction but the yield and ease of reaction is in the order TBPB > TBAB $>$ TBAHS $>$ CTMAB. This may be due to the changes in combination of alkyl groups or may be due to the difference in the activity of anions for phase transfer. The primary requirement of the alkyl groups in PT catalysts is that they collectively should have sufficient organic structure to transfer the desired anion into the organic phase. A catalyst, which is soluble in organic phase, will be better in phase transfer catalysed reactions involving anion transfer from aqueous to organic phase. The larger alkyl groups with almost symmetric structure are most effective both in anion transfer and in reactivity. Quaternary ammonium and phosphonium salts have been successfully used as PT catalysts. Commercially, ammonium salts are more widely available in a large variety of organic structures and are less expensive. Phosphonium salts are thermally stable up to 150 $170{ }^{\circ} \mathrm{C}$, where as ammonium salts lose their activity at temperature greater than $110{ }^{\circ} \mathrm{C}$. The activity of a quaternary salt as a PT catalyst

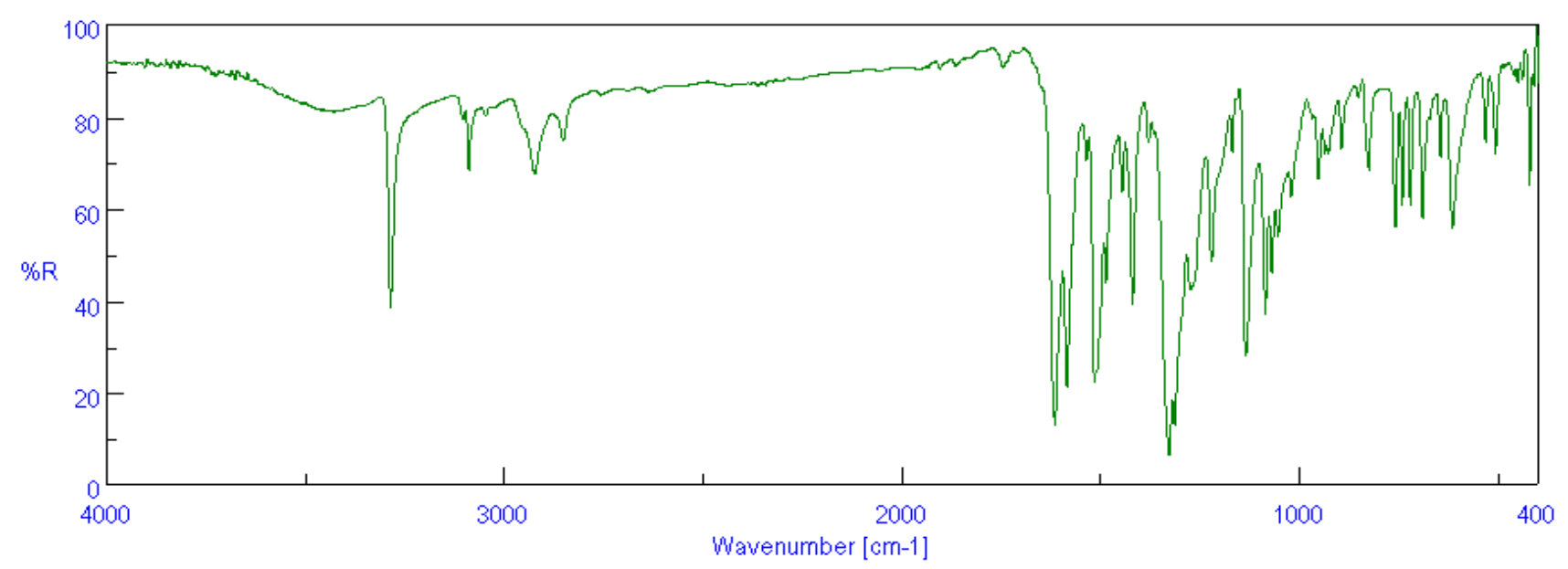

Figure 1. IR spectrum of 2,4-dinitrophenylhydrazone of product on the oxidation of benzyl alcohol. 
depends on the anion originally present. The quaternary salts are useful as PT catalysts only if the anion accompanying the catalyst is distributed in the organic phase to a much lesser extent than the anion to be reacted. In general, the large, lipophilic quaternary cations are soft in the HSAB concept, so that this cation tends to pair with the softest anions available in the solution and transfer it into the organic phase. The more commonly used catalysts have chloride, bromide or hydrogen sulphate as the anion moiety. These facts can be used to explain the order of reactivity of various PT catalysts. The mechanism of the reaction is given in Scheme 1.

\section{Conclusions}

In summary, a simple, efficient, selective green method for the conversion of benzyl alcohols to corresponding benzaldehydes under phase transfer catalysis was reported. Hypochlorite was found to be very effective selective oxidant in the oxidation of benzyl alcohols under PTC. Greener solvents were used for this conversion and there was no formation of by- products. So this method under PTC can be adapted to other reaction systems also and for such reactions under solvent free conditions.

\section{References}

[1] Lee, D.G. (1980). Oxidation of organic compounds by permanganate ion and hexavalent chromium: Open Court: La Salle.

[2] Wiberg, K.B. (1965). Oxidation in organic chemistry: Academic Press, London and New York.

[3] Starks, C.M., Liotta, C. (1978). Phase transfer catalysis, Principles and Techniques: Academic Press, New York.

[4] Dehmlow, E.V., Dehmlow, S.S. (1993). Phase transfer catalysis: VCH: Weinheim, Germany.

[5] Sasson, Y., Neumann, R. (1993). Handbook of Phase Transfer Catalysis: Kluwer Academic Publishers, Dordrecht.

[6] Pletcher, D., Tait, S.J.D. (1979). A procedure for the oxidation of alcohols to aldehydes based on phase transfer catalysis. Journal of the Chemical Society, Perkin Transactions, 2(6): 788-791.

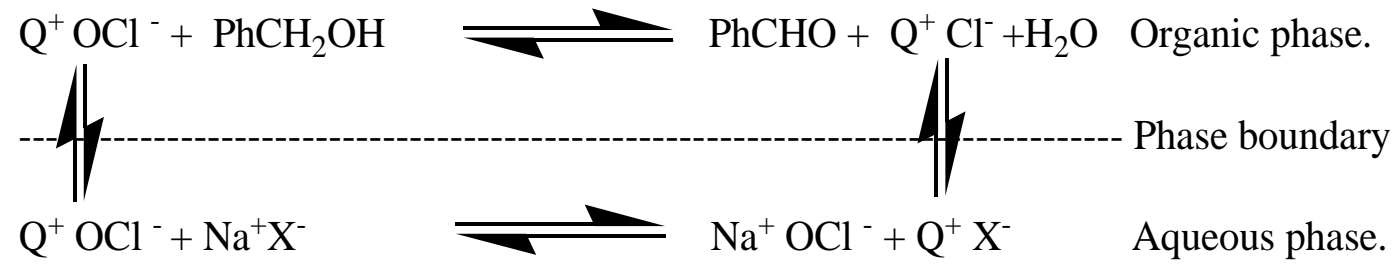

Scheme 1. Oxidative conversion of benzyl alcohols to corresponding benzaldehydes

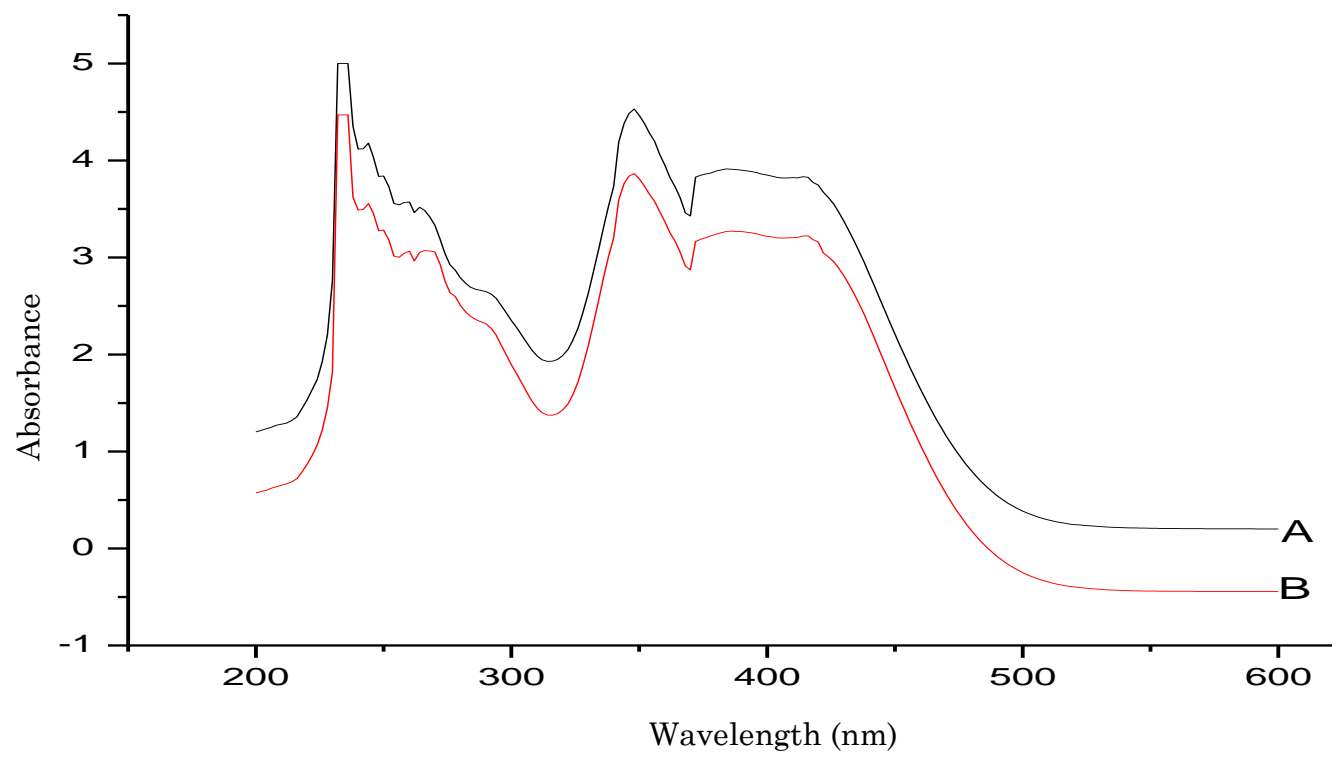

Figure 2. UV-Visible spectrum of (A): 2,4-dinitrophenylhydrazone of pure benzaldehyde; (B): 2,4- dinitrophenylhydrazone of product on the oxidation of benzyl alcohol 
[7] Yadav, G.D., Mistry, C.K. (2001). Oxidation of benzyl alcohol under a synergism of phase transfer catalysis and heteropoly acids. Journal of Molecular Catalysis A, 172(1-2): 135149.

[8] Bijudas, K., Radhakrishnan Nair, T.D. (2004). Selective oxidation of benzyl alcohol with monochromate in non - polar solvents. Indian Journal of Chemistry, 43A: 1216-1218.

[9] Bijudas, K., Radhakrishnan Nair, T.D. (2014). Kinetics and mechanism of the selective oxidation of primary aliphatic alcohols under phase transfer catalysis. Current Chemistry Letters. 3(2): 109-114. (DOI:10.5267/ j.ccl.2013.12.001).

[10] Basheer, K.M., Joseph, J., Radhakrishnan Nair, T.D. (2013). Kinetics of the oxidation of benzhydrols with permanganate under phase transfer catalysis in organic solvents. Modern Research in Catalysis, 2: 35-38.

[11] Bijudas, K., Bashpa, P., Radhakrishnan Nair T.D. (2014). Kinetic studies on the selective oxidation of benzyl alcohol and substituted benzyl alcohols in organic medium under phase transfer catalysis. Bulletin of Chemical Reaction Engineering \& Catalysis, 9(2): 142147. (DOI: 10.9767/bcrec.9.2.6476.142-147).
[12] Wang, M.L., Huang, T.H. (2007). Kinetic study of benzyl alcohol oxidation under phase transfer catalysis conditions, Chemical Engineering Communications, 5: 618-634. (DOI: 10.1080/00986440600992685)

[13] Abramovici, S., Neumann, R., Sasson, Y. (1985). Sodium hypochlorite as oxidant in phase transfer catalytic systems. Part II. Oxidation of aromatic alcohols. Journal of $\mathrm{Mo}$ lecular Catalysis, 29: 299-303.

[14] Mirafzal, G.A., Lozeva, A.M. (1998). Phase transfer catalysed oxidation of alcohols with sodium hypochlorite. Tetrahedron Letters, 39(40): 7263-7266.

[15] Lee, G.A, Freedman, H.H. (1985). Phase transfer oxidations with hypochlorite: Scope and mechanism. Israel Journal of Chemistry, 26: 229-234.

[16] Do, J.S., Chou, T.C. (1990). Kinetics of the oxidation of benzyl alcohol by hypochlorite ion in the presence of phase transfer catalyst. Industrial \& Engineering Chemistry Research, 29: 1095-1103.

[17] Amsterdamsky, C. (1996). PTC Oxidation of alcohols using hydrogen peroxide. Journal of Chemical Education, 78: 66-67.

[18] Vogel, A.I. (1967). Text book of practical organic chemistry: Longman, London. 